\title{
Investigation of the steady and unsteady forces acting on a pair of circular cylinders in crossflow up to ultra-high Reynolds numbers
}

\author{
Günter Schewe ${ }^{1} \cdot$ Nils Paul van Hinsberg ${ }^{2}$ D Markus Jacobs $^{3}$
}

Received: 30 January 2021 / Revised: 16 July 2021 / Accepted: 18 July 2021 / Published online: 29 July 2021

(c) The Author(s) 2021

\begin{abstract}
Measurements of the steady and unsteady forces acting on a pair of circular cylinders in crossflow are performed from subcritical up to ultra-high Reynolds numbers. The two cylinders with equal diameters $d$ are arranged inline at two centre-tocentre distances: $S / d=2.8$ and 4 . The trend of the drag curve for the upstream cylinder $C d_{1}(R e)$ at both distances is similar to that for a single circular cylinder. The development of the drag curves $C d_{2}(R e, S / d=2.8,4)$ of the downstream cylinder is inverse to that of the upstream cylinder. For both cylinder spacing values, the drag on the downstream cylinder is negative for subcritical Reynolds numbers, increases abruptly to positive values at the beginning of the supercritical regime, and shows a significant dip at transcritical Reynolds numbers. This drag inversion indicates that the critical distance $S c$ decreases sharply in the supercritical Reynolds number range. For $S / d=2.8$ at $R e \rightarrow 10^{7}$, the downstream cylinder experiences once more a thrust force. The curve of the Strouhal number $S t(R e)$ of the downstream cylinder for $S / d=4$ is very close to that of a single cylinder. For Reynolds numbers of $R e \approx 1 \times 10^{6}-7 \times 10^{6}$, the Strouhal numbers have nearly equal values of $S t \approx 0.22-0.24$ for both distances. This is followed by a branching. For $R e \rightarrow 10^{7}$ and the case $S / d=2.8$, the Strouhal numbers dip at $S t=$ 0.17 . However, for $S / d=4$, they increase up to $S t=0.27$. In the supercritical range, two peaks occur in the power spectra for the large distance $S / d=4$. Based on a wavelet analysis, we can conclude that the low-frequency mode, which does not occur for a single cylinder, is an interference effect.
\end{abstract}

Nils Paul van Hinsberg

nils.vanhinsberg@dlr.de

Günter Schewe

guenter.schewe@dlr.de

1 Institute of Aeroelasticity, German Aerospace Center (DLR),

Bunsenstr. 10, Göttingen 37073, Germany

2 Institute of Aeroelasticity, German Aerospace Center (DLR),

Göttingen, Germany

3 German Aerospace Center (DLR), Göttingen, Germany 


\section{Graphic abstract}

subcritical $\operatorname{Re} \approx 10^{5}$

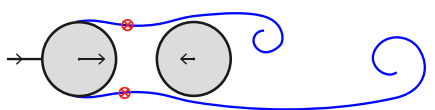

supercritical $\operatorname{Re} \approx 4 \times 10^{5}$

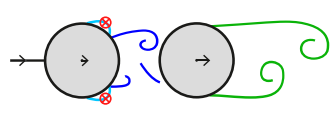

transcritical $\operatorname{Re} \approx 10^{7}$

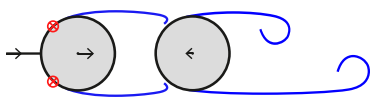

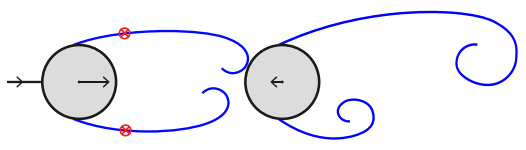
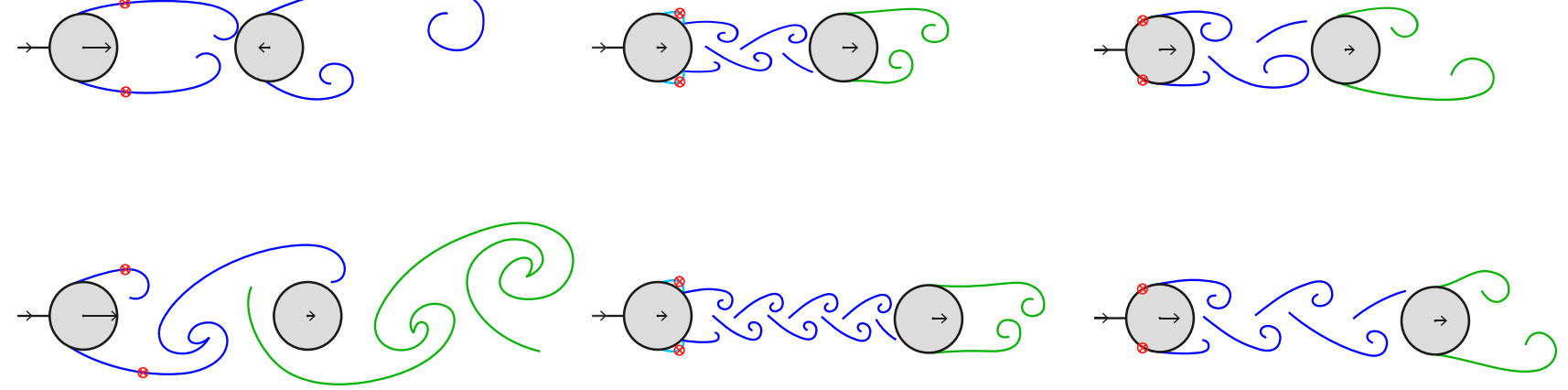

Qlam. / turb. transition

\section{Topological changes depending on the Reynolds number and spacing}

\section{Introduction}

The flow around a pair of circular cylinders is the paradigm example for the study of interference effects in fluid mechanics. Even with a single cylinder, there are very strong Reynolds number effects, and the situation becomes even more complex when a second circular cylinder is placed in the vicinity. The current study concentrates on the "simple" case where the two cylinders are arranged inline. The Reynolds number is varied over three orders of magnitude for two relevant cylinders' centre-to-centre distances. Apart from the measurement of the mean forces and mean base pressures on both cylinders, this study focuses on the measurement and analysis of the fluctuating forces acting on the downstream cylinder. In this way, we obtain information on the spectra, Strouhal numbers, and RMS values of the lift forces. In addition, we conduct a wavelet analysis on the data to obtain information on the time-frequency behaviour of the phenomena.

(Sumner 2010) carried out a review of experimental and numerical studies on the flow around two circular tandem cylinders. Based on the investigations discussed, the interference between the two individual cylinders has a strong influence on the flow topology around the tandem arrangement. In particular, the centre-to-centre spacing $S$ and the Reynolds number determine whether the two cylinders behave as one extended bluff body or as two clearly separated bodies.

If $S$ is small, negative drag forces can occur on the downstream cylinder (Kuzniecow (1931); Hoerner (1958)). With increasing $S$, a jump to positive drag forces appears, i.e. a drag inversion. The location of the sign reversal is called the critical spacing $S c$. For subcritical Reynolds numbers, these phenomena can be explained by the existence of different modes or states of the flow topology and vortex shedding. (Zdravkovich 1987) and (Alam et al. 2018) characterized the different states as follows: there are three basic types of the flow structure possible, namely "extended-body regime", "reattachment regime" and "co-shedding regime". In the first regime, i.e. for $1.0<S / d<1.5$, both cylinders are close together such that the shear layers that have separated from the upstream cylinder can overshoot the downstream cylinder and the flow in the gap is rather stagnant. For an increased distance of $1.5<S / d<3.5$, the second regime is 
present, in which the free shear layers that separate from the upstream cylinder can reattach to the downstream cylinder. Additionally, for these distances, the flow in the gap remains almost stagnant. In both cases, proximity effects dominate, and one common vortex street is formed behind the downstream cylinder. Both cases are denoted as "mode I". In other words, the "extended-body flow" and "reattachment flow" are a subdivision of "mode I", i.e. the state in which proximity interference is dominant. At large spacing, i.e. for $S / d>3.5$, the separated free shear layers from the upstream cylinder are able to form vortical structures, which travel downstream before impinging on the downstream cylinder. In this "co-shedding regime" or "mode II", both cylinders shed vortices. The value $S / d \approx 3.5$ at which the transition between both modes occurs is denoted as the "critical spacing" Sc. Notably, around $S c$, bistable states can occur, in which the flow jumps intermittently between the "reattachment" and the "co-shedding" modes. This is a typical behaviour of such transition phenomena in fluid flow. We present and discuss various simplified sketches of the different flow states later with Fig. 12.

For a tandem arrangement with a small centre-to-centre distance of $S / d=1.56$, experiments by (Schewe and Jacobs 2019) showed that the critical spacing $S c$ depends on the Reynolds number range and that a drag inversion is an indication for a change in the modes from a state in which proximity interference dominates to a "co-shedding flow" or vice versa. Regarding the subcritical case, our present experiments were performed for two distances, namely below ( $S / d$ $=2.8)$ and just above the critical value $(S / d=4)$.

For very high Reynolds numbers, only a few investigations are available. For two distances $S / d=3$ and 5, the results from (Pearcy et al. 1982), obtained at high Reynolds numbers, can be found in (Zdravkovich 1987). Their data include drag coefficients and Strouhal numbers up to supercritical Reynolds numbers of $R e \approx 7 \times 10^{5}$ and up to $R e=7 \times$ $10^{6}$ for $S / d=3$ and 5, respectively. For one typical subcritical Reynolds number, (Alam et al. 2003) performed extensive experiments in which the spacing was systematically changed in small steps in the range of $1.1 \leq S / d \leq 9$. Their results show that at a spacing of $S / d=4$, sudden jumps in the drag coefficient and the Strouhal number appear, both of which are caused by the transition from "mode I" to "mode II". Consequently, in their studies, the critical spacing has a value of $S c / d=4$. Regarding our values obtained in the subcritical Reynolds number range, we use their results for comparison.

(Schewe and Jacobs 2019) performed experiments for a large Reynolds number range from $R e=2 \times 10^{5}$ up to $R e$ $=6 \times 10^{6}$ for a cylinder centre-to-centre spacing of $S / d=$ 1.56. They measured the steady aerodynamic forces and investigated the influence of the angle of incidence and the propensity to flow-induced vibrations. Among others, their results show that for the inline configuration, the appearance of the drag curve for the upstream cylinder is similar to that of a single smooth circular cylinder. They therefore used the same classification for the individual Reynolds number ranges, i.e. sub-, super-, and transcritical. In the small critical Reynolds number regime at approximately $R e_{c}=3 \times 10^{5}$, the transition from the sub- to the supercritical range occurs, the latter extending up to $R e \approx 10^{6}$. After a second, rather long transition regime, a new stable state, the transcritical Reynolds number range, is reached at approximately $R e=$ $5 \times 10^{6}$.

The present measurements have been obtained in the same high-pressure wind tunnel in which the studies by (Schewe 1983) and (Schewe and Jacobs 2019) were conducted. The mean and fluctuating forces on the downstream cylinder were measured with a rigid piezo-balance. The Reynolds number was varied between $R e=7 \times 10^{4}$ and $1.2 \times$ $10^{7}$. The desired maximum Reynolds number was achieved without any compromises regarding the size of the model, Mach number effects $(M<0.1)$, geometric blockage effects $(10 \%)$, surface roughness, or inflow turbulence intensity.

\section{Experimental arrangement}

\subsection{Test set-up}

As mentioned before, the current experiments were carried out in the High-pressure wind tunnel of the DLR Institute of Aeroelasticity. The wind tunnel is of the closed return type and can be pressurized up to $10 \mathrm{MPa}(100 \mathrm{bar})$. In that way, maximal Reynolds numbers on the order of $10^{7} \mathrm{can}$ be achieved in an incompressible flow of $M \leq 0.1$. The main details of the wind tunnel are as follows:

- maximum flow speed: $U=35 \mathrm{~m} / \mathrm{s}$;

- size of square test section: $0.6 \times 0.6 \mathrm{~m}^{2}$ :

- total pressure range: $1 \leq p_{0} \leq 100$ bar;

- Reynolds number range: $10^{4}<R e<10^{7}$, based on the cylinder dimension $d=0.06 \mathrm{~m}$.

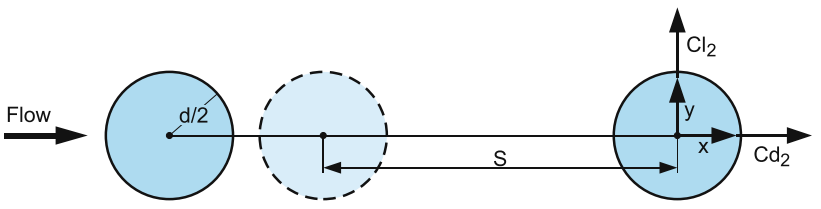

Fig. 1 Coordinate system of cylinder arrangement. In the present experiments, the forces on the downstream cylinder were measured by a piezo-balance. The distance $S$ between the cylinders - the centreto-centre spacing-is $S / d=2.8$ and 4 
The closed test section is $1 \mathrm{~m}$ long and can be decoupled from the wind tunnel by means of an air lock system, while the tunnel tube is kept under pressure. The drag and lift forces on the downstream cylinder were obtained using piezo-balances, described in detail in (Schewe 2007). The resultant mean drag and lift coefficients are defined as $C d_{2}$ $=F_{x} /(q L d)$ and $C l_{2}=F_{y} /(q L d)$, respectively, where $F_{x}$ is the drag force, $F_{y}$ is the lift force, $q$ is the dynamic pressure, and $L$ is the span of the section. The subscript "2" denotes the downstream cylinder. The forces were measured in the fixed wind tunnel coordinate system, as displayed in Fig. 1.

The cylinder diameter is $\mathrm{d}=0.06 \mathrm{~m}$, resulting in a geometric blockage of $10 \%$. The distance $S$ between both cylinders, i.e. the centre-to-centre spacing, is either $S / d=2.8$ or 4. The surface roughness of each cylinder equals $k / d=3 \times$ $10^{-6}$, meaning that both cylinders can be classified as very smooth. The aspect ratio of the cylinders with span $L$ is relatively high: $d: L=1: 10$. The lowest eigenfrequencies of the downstream cylinder are related to its bending modes in the stiff piezo-balance, $f_{z}=489 \mathrm{~Hz}$ in the lift direction and $f_{x}=435 \mathrm{~Hz}$ in the drag direction. Typical vortex shedding frequencies are less than one-third of the structural eigenfrequencies. An extreme case taken at the highest possible total air pressure of $100 \mathrm{bar}$ and at $R e=1.1 \times 10^{7}$ is shown in Figs. 10c, 11. For the calculation of the RMS values, the contribution of the structural bending was extracted by integrating the corresponding PSD up to $280 \mathrm{~Hz}$ only. In most cases, the contribution of bending motion is negligible

A wake rake was applied to determine the mean pressure distribution in the combined wake of both cylinders. In a post-processing step, these data were then used to calculate the sectional total drag coefficient $C d_{\text {rake }}$. The pressure rake was located at the midspan position, close to the exit of the test section. The distance from the centre of rotation of the downstream cylinder (attached in the balance) to the tips of the Pitot probes was $390 \mathrm{~mm}$, which corresponds to $6.5 d$. The wake rake was equipped with 6 static pressure tubes and 52 Pitot pressure tubes, both distributed symmetrically around the centreline. Near the centreline, the distances between the Pitot pressure probes were $\Delta z=6 \mathrm{~mm}$. The distance $\Delta z$ increases when $z$ approaches the upper or lower wall. For all measurements, the signals were acquired with a sample rate of $F_{s}=5 \mathrm{kHz}$, an integration time of $T=30 \mathrm{~s}$, and a resolution of 16 bit.

\subsection{Drag measurement}

As briefly discussed in the preceding section, we used two methods for the drag measurements: the wake rake for obtaining the total drag on the tandem arrangement $C d_{\text {rake }}$ and the piezo-balance for the measurement of the steady and fluctuating forces $C d_{2}(t)$ acting only on the downstream cylinder. We can thus obtain information on the mean drag force on the upstream cylinder by forming the difference $C d_{\text {rake }}-C d_{2}=C d_{1}$. The accuracy of $C d_{1}$ is reduced because of a principal problem: the balance measures the global force on the entire cylinder, i.e. it integrates in the spanwise direction from wall to wall, while the rake force measurement is a sectional one where it is assumed that the flow around the body is two-dimensional. In particular, in the transition regions, the flow around nominally two-dimensional bluff bodies can nevertheless be substantially three-dimensional and is accompanied by the formation of span wise cell-like structures (Schewe 2001). The results can thus also depend on the actual position of the individual cell structures in relation to the location of the sectional measurement. We are aware that this procedure is not ideal, but this method is the only one available that keeps the experimental effort within reasonable limits.

\section{Experimental results}

\subsection{Drag coefficients}

The measurements were performed for two distances $S / d=$ 2.8 and 4 over a large range of Reynolds numbers of $7 \times 10^{4} \leq$ $R e \leq 1.2 \times 10^{7}$. As mentioned in the introduction, (Alam et al. 2003) provided a large set of measured quantities, among which characteristic values are the Strouhal number and RMS values. Regarding the subcritical Reynolds number range, we use their results for comparison.

The measured force coefficients have not been corrected for wall interference effects since there is no valid correction method for such a large Reynolds number range in which the separation location moves substantially. Furthermore, we know of no method to correct the unsteady measurements. In (Schewe 1983), these points were already discussed in detail for a single circular cylinder with an equal geometric blockage ratio of $10 \%$.

In Fig. 2, the total drag of the tandem arrangement, obtained from the pressure rake in the wake behind both cylinders, is plotted as a function of the Reynolds number. Examples of various wake profiles for both distances $S / d$ are given in Fig. 3. For comparison reasons, the curve of the drag coefficient $C_{D}(R e)$ of a single smooth circular cylinder by (Schewe 1983) is included in Fig. 2. The latter data were obtained using a piezo-balance.

At first glance, the appearances of both $C d_{\text {rake }}(R e)$-curves for the tandem cylinder arrangement are similar to the corresponding curve of a single smooth circular cylinder with $S / d$ $=0$. However, in the case of $S / d=4$, the drag coefficients in the individual ranges are much higher than those of a single circular cylinder. The reason for this large difference in drag force is discussed in sect. 4. For a single smooth cylinder, the drag coefficients are, for example, approximately 1.2 
Fig. 2 Total mean drag coefficient $C d_{\text {rake }}$ of the tandem arrangement as function of the Reynolds number. The mean drag forces were obtained using a pressure rake behind the downstream cylinder, whereas the mean drag coefficients $C_{D}$ of a single circular cylinder $(\bullet)$ were obtained with a piezobalance (Schewe 1983)

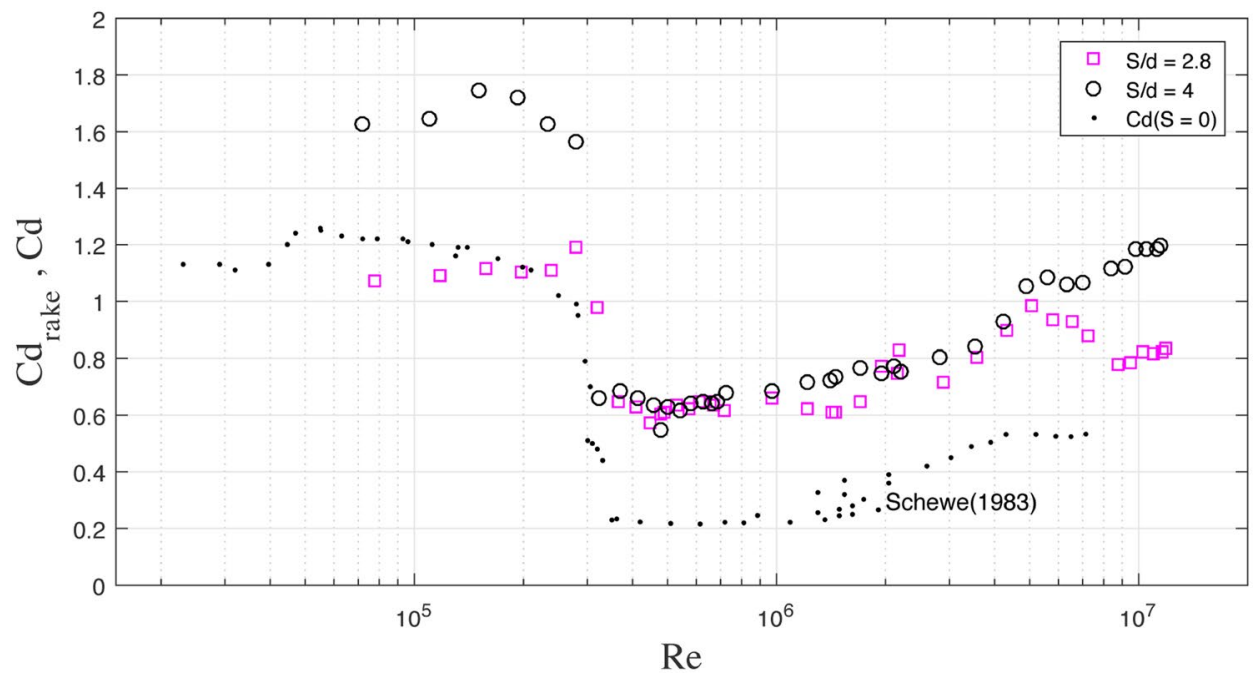

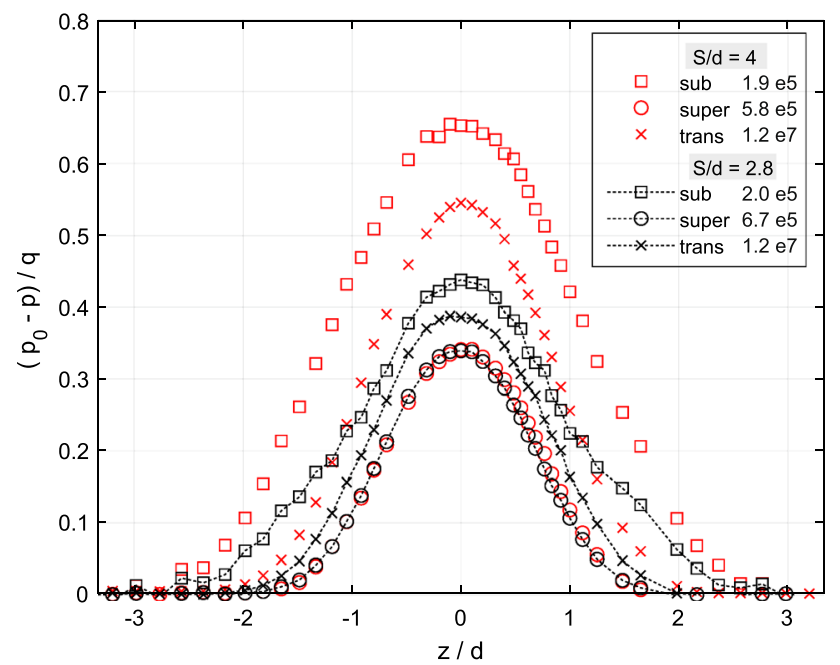

Fig. 3 Typical mean wake profiles for each Reynolds number range $\left(z / d\right.$ : vertical position/cylinder diameter; $p$ : pressure at location $z ; p_{0}$ : total pressure; $q$ : dynamic pressure)

in the subcritical range, 0.2 in the supercritical range, and 0.5 in the transcritical Reynolds number range. The location of the critical Reynolds number regime at $3.5 \times 10^{5}$ then again nearly coincides with that of a single circular cylinder. A long supercritical range up to $R e \approx 10^{6}$ is present for both distances, where the drag coefficient lies in both cases around $C d_{\text {rake }} \approx 0.6$. After a second, extended increase in both drag curves up to approximately $R e=5 \times 10^{6}$ to $6 \times 10^{6}$, the $C d_{\text {rake }}$-curve for $S / d=4$ exhibits a very small local maximum that is followed by a further increase in the total drag. For the smaller distance $S / d=2.8$, there is a pronounced maximum with $C d_{\text {rake }} \approx 1$ at $R e=5 \times 10^{6}$, followed by a steady decrease to 0.8 for Reynolds numbers approaching $R e$ $\rightarrow 10^{7}$. The reason for this decline in total drag is the sharp drag force reduction that acts on the second cylinder $C d_{2}$ at approximately $R e=10^{7}$.

As mentioned before, Fig. 3 presents typical wake profiles for both distances in each Reynolds number range. The integrated area corresponds to the sectional total mean drag coefficient $C d_{\text {rake }}$ of the tandem arrangement. The width of the distribution is a measure of the width of the wake. At the subcritical Reynolds number $R e=1.9 \times 10^{5}$, the distribution exhibits the largest width for the distance $S / d=4$ ( $\square$ in Fig. 3). In contrast to the measurements for $S / d=1.56$ by (Schewe and Jacobs 2019), the distribution of the current data has only one maximum in the subcritical region, whereas for the former distance, two maxima, at $z / d \approx \pm 1$, are present. The reason could be that for the case $S / d=$ 1.56 , the flow is in the "elongated body mode" (the first subdivision of "mode I"), for the present case $S / d=2.8$ in the "reattachment mode" (second subdivision of "mode I"), and for $S / d=4$ in the "co-shedding mode", i.e. "mode II". Furthermore, for the case $S / d=1.56$, the distance from the centreline of the downstream cylinder to the wake rake is $10.3 d$, whereas for $S / d=2.8$ and 4 , it is only $6.5 d$.

As expected, we have the smallest area and the smallest width in the supercritical case (o/o in Fig. 3) for both distances $S$. The curves are practically identical, which corresponds to the drag coefficients in the supercritical range presented in Fig. 2. In the transcritical range ( $\times / \times$ in Fig. 3), the values for the wake width and the area lie between both Reynolds number cases mentioned above, whereby the values for the distance $S / d=4$ are found to be somewhat higher.

Figure 4a, b displays the individual mean drag forces $C d_{1}$ $(R e)$ and $C d_{2}(R e)$ for distances $S / d=2.8$ and 4.0. We recall once more that the mean drag force on the upstream cylinder, $C d_{1}$, is given by the difference between the total mean drag coefficient $C d_{\text {rake }}$ of the tandem arrangement and the mean drag coefficient that acts on the downstream cylinder $C d_{2}$. To show the development from smaller to larger spacing $S / d$ 

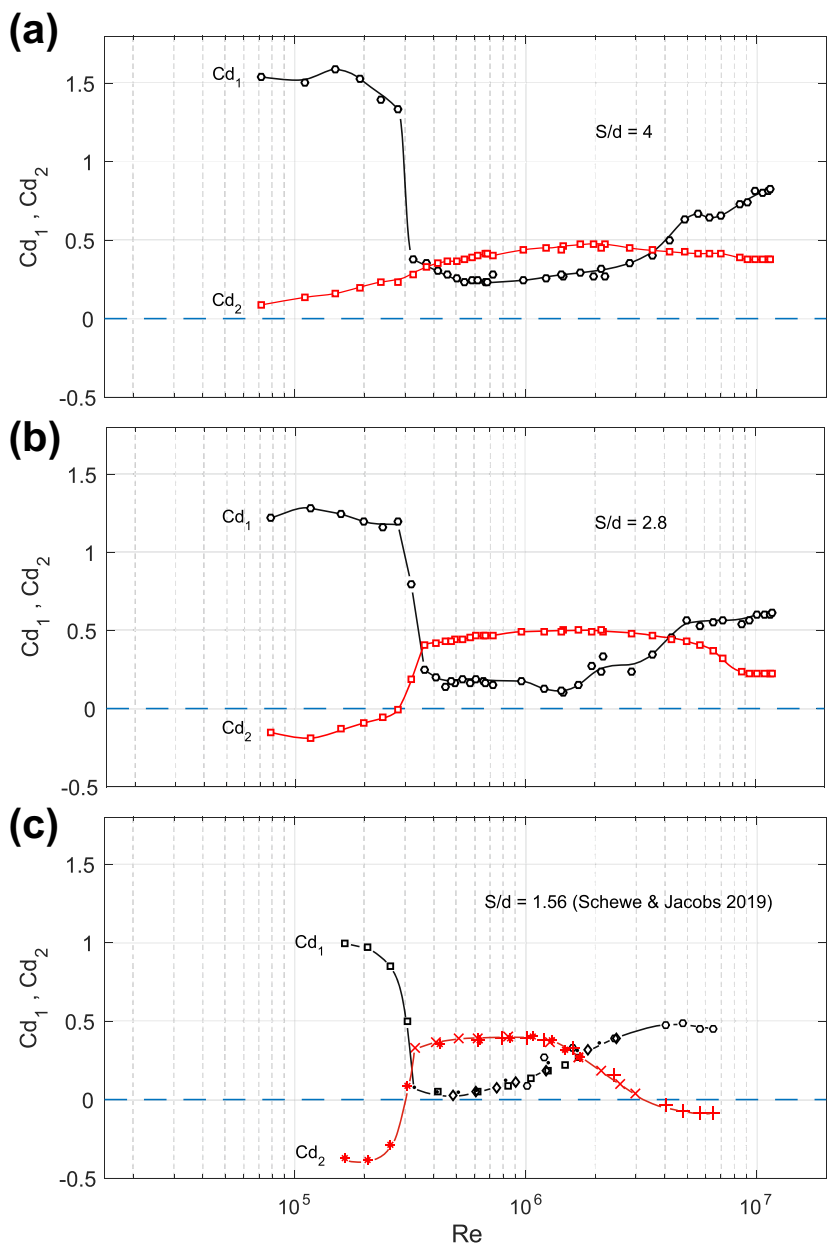

Fig. 4 Mean drag coefficients for both individual cylinders as function of the Reynolds number for three spacing values $S / d=1.56,2.8$, and 4.0. In Fig. 4c the different symbols represent different total air pressures: $\square / *$ equals 20 bar, $\bullet / \times$ equals 40 bar, $\diamond$ equals 60 bar, $\square /+$ equals 80 bar

in Fig. 4, the corresponding figure of (Schewe and Jacobs 2019) is included in Fig. 4c. The different symbols in both curves in Fig. $4 \mathrm{c}$ represent different total air pressures $p_{0}$ in the High-pressure wind tunnel. The latter measurements were performed in the same wind tunnel for a significantly smaller distance of $S / d=1.56$. In contrast to the current measurements, at $S / d=1.56$, only steady forces on both cylinders were determined by means of a strain gauge balance.

For all three cases $S / d=1.56,2.8$, and 4 , the appearance of the curves for the upstream cylinder $C d_{1}(R e)$ is similar to the behaviour of the total drag shown in Fig. 2 and of a single smooth circular cylinder $(S / d=0)$. As the curves of $C d_{1}(R e)$ in Fig. 4 a, b are partly based on wake drag measurements that are local, i.e. sectional, the scatter of the individual data is higher than in the case of a pure integrating balance measurement for $C d_{2}(R e)$, as displayed in Fig. $4 \mathrm{c}$ for $S / d=1.56$.
At equal Reynolds numbers, the values of the drag coefficients $C d_{1}$ in the individual ranges are lower for smaller distances $S / d$. Beyond the critical Reynolds number regime for $S / d=1.56$, there is a long supercritical range up to approximately $R e \approx 10^{6}$. For both larger distances $S / d=$ 2.8 and 4.0 , this regime is longer and extends up to $R e \approx 2 \times$ $10^{6}$. Considering the cases $S / d=1.56$ and 2.8 in Fig. $4 \mathrm{c}$, b, respectively, a second plateau for $C d_{1}$ is reached at approximately $\operatorname{Re}(S / d=1.56) \approx 5 \times 10^{6}$ and $\operatorname{Re}(S / d=2.8) \approx 6 \times 10^{6}$, respectively, preceded by a second, rather long continuous increase in $C d_{1}$.

The individual shapes of the $C d_{2}(R e, S / d=1.56 ; 2.8 ; 4)$ curves of the downstream cylinder reveal an inverse development with the Reynolds number compared to the upstream ones. For $S / d=1.56$, during the transition from sub- to supercritical Reynolds numbers, a sharp change occurs in the drag coefficient from approximately $C d_{2} \approx-0.3$ to $C d_{2} \approx$ +0.4 (supercritical), i.e. exceeding zero. For the larger distance $S / d=2.8$, this change is less prominent, from approximately $C d_{2} \approx 0$ (subcritical) to $C d_{2} \approx 0.4$ (supercritical). This small transition range around $R e_{c} \approx 4 \times 10^{5}$ is the critical Reynolds number regime.

In the subcritical regime, the negative sign of the mean drag force on the downstream cylinder indicates that the spacings $S / d=1.56$ and 2.8 are below the critical spacing $S c$; thus, the state of the flow around both cylinders belongs to the "reattachment regime" ("mode I"), where a vortex street is formed behind the downstream cylinder only. For the largest spacing $S / d=4$, however, no zero-crossing of the downstream cylinder's mean drag force is found. In the subcritical state, the drag is indeed small but positive $\left(\mathrm{Cd}_{2}\right.$ $\approx+0.08$ ), leading to the conclusion that the state of the flow is in the "co-shedding mode" ("mode II"). The character of the drag curve $C d_{2}(R e)$ is quite different from that of both other curves; the variations are rather small, and there is no discontinuous jump of the drag force in the critical regime. One probable reason is the nonexistence of a change in the modes, meaning that the distance is too large for impactful proximity interference effects.

However, a common property of all three distances is the paradox situation, in which the mean drag on the downstream cylinder in the supercritical range is higher than that on the upstream cylinder. The reason for the phenomena that take place in the supercritical regime is the formation of separation bubbles on the cylinder's surface in the critical state ( (Zdravkovich 1987)). Following the classic paper by (Roshko 1961) regarding a single circular cylinder, the key role is played by the location of the laminar/turbulent transition in the separated shear layers. At subcritical Reynolds numbers, the transition occurs in the wake behind the cylinder. With increasing Reynolds number, the transition location shifts upstream. In the critical range, the transition has reached the shoulders of the upstream cylinder. Separation 
bubbles can thus be formed on its surface, leading to a sharp reduction in the wake width of the upstream cylinder. Experimental evidence of separation bubbles in the supercritical state can be found in the surface pressure distribution at $R e$ $=6.7 \times 10^{5}$ measured by (Flachsbart 1929) and reproduced by (Roshko 1961). The occurrence of separation bubbles at $R e=6.5 \times 10^{5}$ was also confirmed through an LES simulation by (Rodriguez et al. 2015). The reduction in the wake width behind the upstream cylinder as a result of the formation of those separation bubbles leads to an increase in the drag on the downstream cylinder $C d_{2}$. These transition effects are strong for both cases when $S<S c$ and weak for $S>S c$. Considering the case $S / d=1.56$, the bubbles remain rather stable up to $R e \approx 10^{6}$. As a consequence, the drag coefficient $C d_{2}$ remains nearly constant at a value of $C d_{2} \approx 0.4$ This is followed by a new transition region that is caused by the continuous disappearance of the separation bubbles at the upstream cylinder, resulting in an increase in the wake width and the drag force. Regarding a single cylinder, (Roshko 1961) named this regime the "upper transition". The opening of the wake of the upstream cylinder is coupled with a continuous decrease in the drag on the downstream cylinder. At approximately $R e \approx 3.5 \times 10^{6}$, a second sign reversal of the drag takes place to values down to $C d_{2} \approx-0.07(<0)$, the transcritical plateau. It can therefore be concluded that the state of the flow has changed again, from the "co-shedding mode" back to "mode I" in which proximity interference effects are dominant. More precisely, a transition to the "reattachment mode" takes place, in which the free shear layers that have separated from the upstream cylinder can reattach to the downstream cylinder.

With regard to the medium distance $S / d=2.8$, the trend of the curve $\mathrm{Cd}_{2}(\mathrm{Re})$ is similar, including a significant decline in $C d_{2}$ when the Reynolds number approaches very high values of $R e \rightarrow 10^{7}$. However, compared to the preceding case, the decline is not as steep and begins later at approximately $R e=3 \times 10^{6}$. In addition, the transcritical plateau around $R e$ $\approx 10^{7}$ remains at a positive value of $C d_{2} \approx 0.2$; hence, there is no zero-crossing, as was found for the smallest distance.

It was already mentioned that for the largest distance $S / d$ $=4$, the behaviour of the drag curve of the downstream cylinder $C d_{2}(R e)$ is in the critical Reynolds number range rather different from the case of both other distances. This is also valid in the very high Reynolds number regime where the decline in the mean drag is relatively small, i.e. from $C d_{2}$ $\left(\operatorname{Re} \approx 2 \times 10^{6}\right) \approx 0.5$ to a value of 0.4 at $R e \approx 10^{7}$. The most obvious difference is the nonexistence of a distinctive dip of the mean drag on the downstream cylinder for $S / d=4$.

The measured mean lift coefficients for the downstream cylinder, which are not shown here, are practically zero over all investigated Reynolds number ranges. If one were to pass through the critical regions in smaller velocity steps than used in the current study, one would most likely find steady

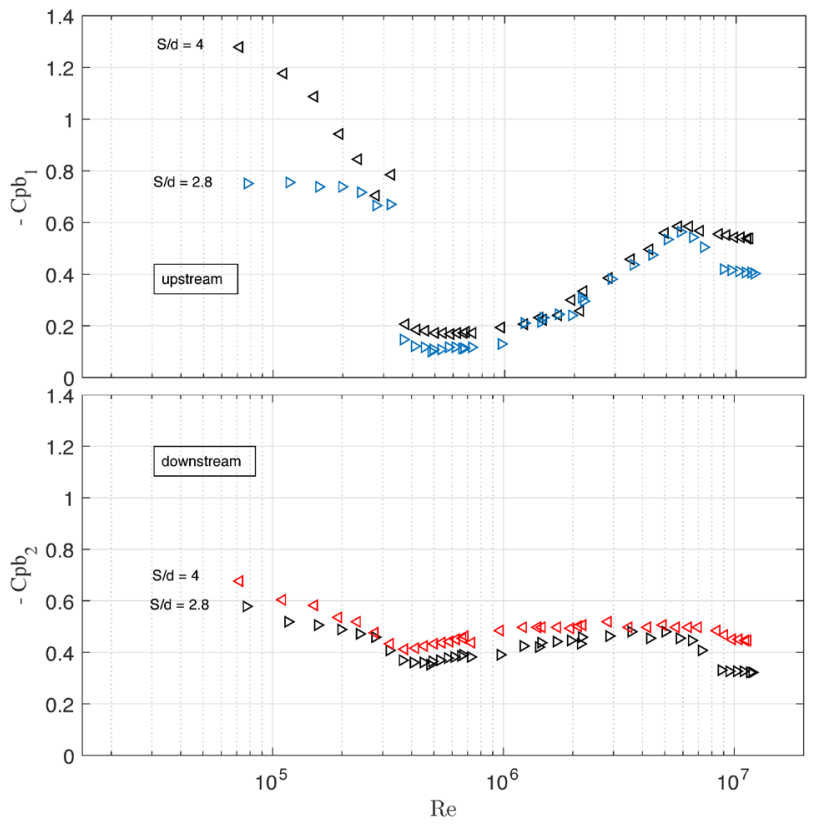

Fig. 5 Mean base pressure coefficients $C p b_{1}$ and $C p b_{2}$ for both cylinders and two spacings $S / d=2.8$ and 4 . The upper figure shows the values for the upstream (index 1) and the lower figure for the downstream cylinder (index 2)

asymmetric states with $\mathrm{Cl}_{2} \neq 0$, as reported in (Schewe 1983) for single smooth cylinder flows and in (Schewe and Jacobs 2019) for two smooth circular cylinders in a tandem arrangement.

\subsection{Base pressure coefficients}

The mean base pressure coefficient $C p b_{1}$ of the upstream cylinder is shown for the distances $S / d=2.8$ and 4 in the upper part of Fig. 5, whereas the lower part of the same figure presents $\mathrm{Cpb}_{2}$ for the downstream cylinder.

Most notable are the trends of both $C p b(R e)$-curves for the upstream cylinder. They both show a similar behaviour as that found for the corresponding drag curves $C d_{1}(R e)$. In the case of the smaller of the shown distances, $S / d=2.8$, there is however a significant dip around $\operatorname{Re} \approx 10^{7}$, i.e. a large increase in the mean base pressure. In particular, in the subcritical region, the absolute values of the mean base pressure of the upstream cylinder are lower for the smaller of the spacing values. The interference effects in the subcritical range, i.e. the occurrence of an inverse drag (hence thrust) on the downstream cylinder is reflected in a significant decrease in the mean suction pressure of $C p b_{1} \approx-0.75$ in comparison to $C p b=-1.2$ for a single cylinder.

A first look at the mean base pressure coefficient of the downstream cylinder, $\mathrm{Cpb}_{2}(\mathrm{Re})$, in the lower part of Fig. 5 confirms a similar trend of both curves as described above for $C p b_{1}(R e)$. A significant difference is the nonexistence of 
a jump in the critical Reynolds number range, though. The variations are furthermore much smaller and at the crossover to the supercritical regime, there appears only a kink in the curve.

Although different values for the cylinder interspacing were chosen, an approximate comparison of the current results with the corresponding measurements of (Alam et al. 2003), taken at a subcritical Reynolds number of $R e=6 \times$ $10^{4}$, can be performed here. (Alam et al. 2003) measured the base pressure of the upstream cylinder of $C p b_{1}=-0.9$ at $S / d$ $=2.2$ and $C p b_{1} \approx-0.68$ at $S / d=3.5$. Our current result of $C p b_{1}=-0.75$ at the distance $S / d=2.8$ in the upper part of Fig. 5 thus lies in an intermediate position. As an example, for a distance with $S>S c$, (Alam et al. 2003) obtained a value of $C p b_{1}=-1.25$ at $S / d=4.5$, which lies close to our result of $C p b_{1}=-1.28$ at $S / d=4$. Regarding the downstream cylinder, the experimental data by (Alam et al. 2003) show a value of $C p b_{2} \approx-0.65$ at $S / d=2.4$, while we obtained $C p b_{2}=-0.58$ at $S / d=2.8$, as presented in the lower part of Fig. 5. For a larger distance beyond the critical value $S c$,
(Alam et al. 2003) measured $C p b_{2}=-0.62$ at $S / d=4.5$, which lies close to our value of $C p b_{2}=-0.68$ at $S / d=4$.

\subsection{Strouhal number and RMS-values of the fluctuating lift forces}

The Strouhal number St and the RMS values of the fluctuating lift coefficient $\mathrm{Cl}_{r m s}$ were obtained from the spectra of the fluctuating lift acting on the downstream cylinder. Their values for spacing $S / d=2.8$ and 4 are plotted in Fig. 6 . The corresponding results for the single smooth cylinder $(S / d=$ 0 ) are also included for comparison. Since the single smooth cylinder had the same diameter $d$ and the measurements were taken in the same wind tunnel with the same balance (Schewe 1983), nearly equal test conditions were obtained in both experiments. Unfortunately, no results for $C l_{r m s}$ and $S t$ are available for the small distance $S / d=1.56$, as presented in the previous figures. The reason behind this is that (Schewe and Jacobs 2019) used a strain gauge balance in their tests, which is not suitable for unsteady measurements.
Fig. 6 RMS-values (upper) and Strouhal numbers (middle and lower figure) obtained from the fluctuating lift forces acting on the downstream cylinder as function of the Reynolds number and the spacing. $\bullet$ : single circular cylinder data by (Schewe 1983). In the supercritical range two peaks occurred in the power spectra at the same Reynolds number. The dominant Strouhal number is indicated by a dot within the circular symbols, whereas the open circles without dots belong to the secondary peak
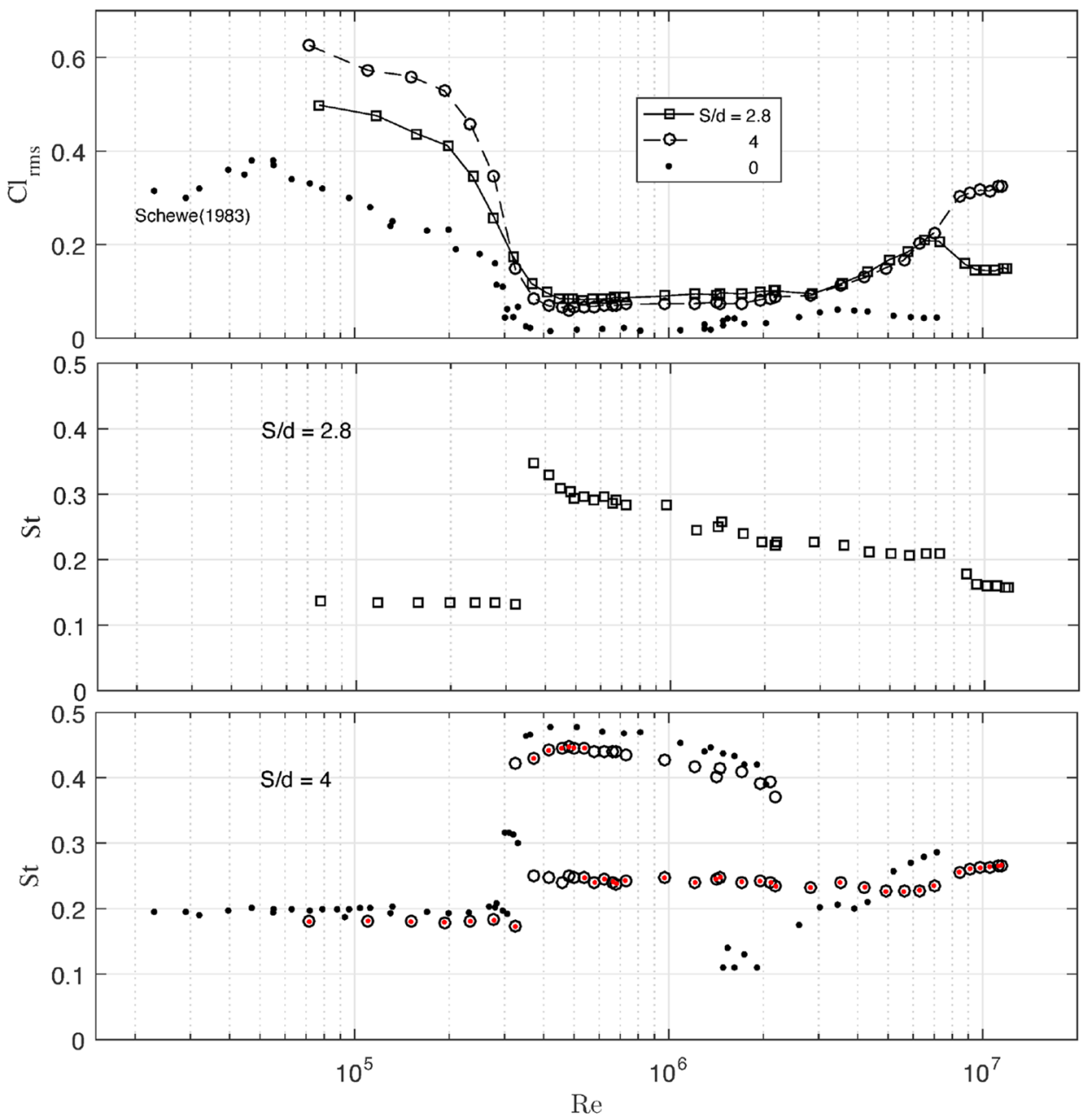
All three plots in Fig. 6 show a strong dependency of the RMS values of the fluctuating lift coefficients and the Strouhal number on the Reynolds number for the downstream cylinder. Note that the precise measurement of force fluctuations is very difficult; however, it is a showpiece of the stiff piezo-balance.

For the sub- and transcritical regimes, the RMS values obtained for the larger spacing $(S / d=4)$ in the upper plot of Fig. 6 are much higher than for the shorter distance $(S / d$ $=2.8$ ). In the transcritical range, the values for $S / d=4$ are even twice as high. In the supercritical regime, the RMS values for the smaller spacing are then again somewhat higher, although the difference is smaller than in both aforementioned regimes. In any case, the RMS values of the fluctuating lift of the downstream cylinder are significantly higher for all studied Reynolds numbers in both tandem arrangements than for a single cylinder. In particular at very high Reynolds numbers near $10^{7}$, these values at the larger spacing are as high as for a single circular cylinder at subcritical Reynolds numbers and approximately 7 times as large as those found for a single circular cylinder at equal transcritical Reynolds numbers.

Considering these results, note that the RMS values of the fluctuating lift are the result of the force fluctuations acting on the entire downstream cylinder that span from one sidewall of the test section to the other; hence, $\mathrm{Cl}_{r m s}$ is in this case related to a cylinder length of $10 \mathrm{~d}$. In comparison, the RMS measurements of the fluctuating lift obtained by (Alam et al. 2003) are, for example, related to cylinder length of $0.92 d$ only. Hence, it can be expected that their RMS values are significantly higher. An interpolation of the measured values in Fig. 13 in (Alam et al. 2003) results in a value of $C l_{r m s} \approx 0.6$ for the smaller spacing $S / d=2.8$, whereas for $S / d$ $=4$, a value of $C l_{r m s} \approx 0.9$ was measured.

In the lower part of Fig. 6, the results for $S t(R e)$ show that for the largest of the three shown spacing values, $S / d$ $=4$, the subcritical Strouhal numbers are approximately $S t$ $=0.18$, close to $S t=0.182$ obtained by (Alam et al. 2003), but approximately $10 \%$ lower than for a single cylinder. At the end of the critical Reynolds number range, there is a jump to the supercritical state. The most striking feature in the supercritical state is the occurrence of two peaks in the power spectra at a constant Reynolds number. In other words, after the jump into the supercritical state, two Strouhal numbers exist at the same Reynolds number. The first peak at $S t=0.44$ is caused by the dominant peak in the spectrum, whereas the second value at $S t=0.24$ results from a secondary peak. Representative examples of the power spectra $\Phi_{C l}(S t)$ are shown in Fig. 7 and are discussed in the next section. The dominant peaks in the spectra are indicated in Fig. 6 by dots within the circular symbols. Hence, open circles without dots belong to secondary peaks. It is evident that the higher frequency component, i.e. $S t \approx 0.4$, dominates

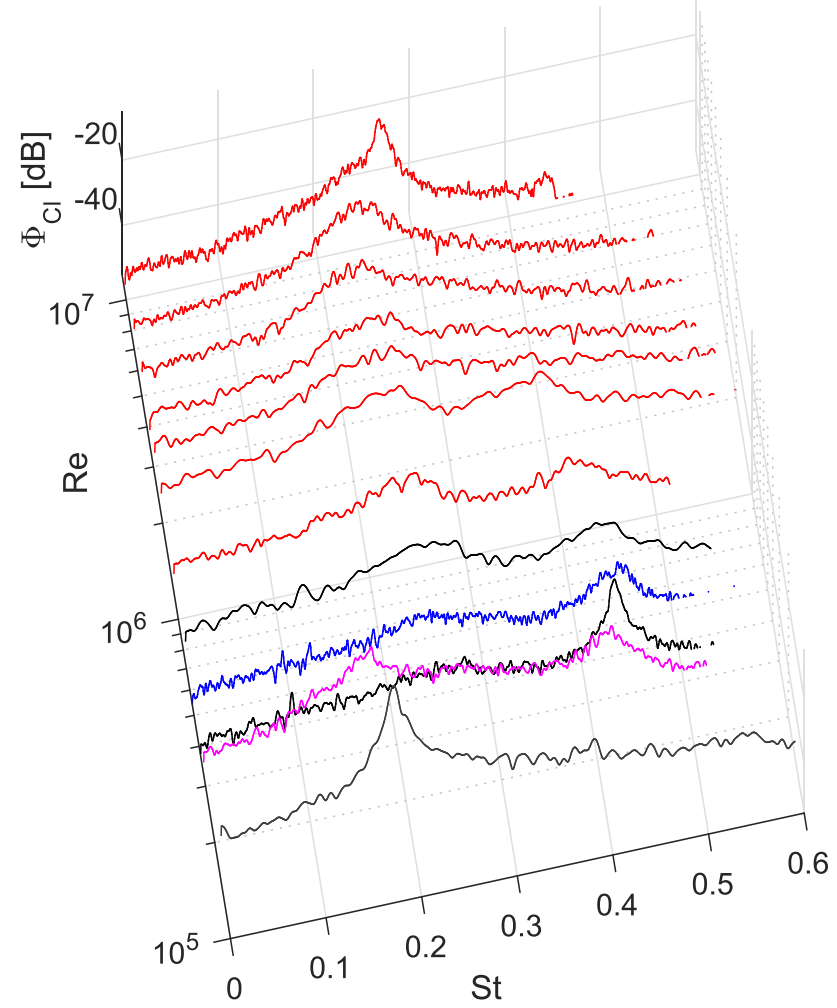

Fig. 7 Development of the power spectra $\Phi_{C l, 2}(S t)$ as a function of the Reynolds number, obtained from the fluctuating lift forces $\mathrm{Cl}_{2}(t)$ that act on the downstream cylinder at a spacing of $S / d=4.0$

up to $R e \approx 5 \times 10^{5}$; thereafter, the lower frequency component predominates, i.e. $S t \approx 0.24$. This behaviour extends up to $R e$ $\approx 2 \times 10^{6}$. If only the high values around $S t \approx 0.4$ are considered, then the shape of the $S t(R e)$-curve is similar to that of the single cylinder, and the values of the respective ranges (super- and transcritical) are approximately 10\% lower. In conclusion, we can state that, apart from the transition range around $R e \approx 10^{6}$, the current measured values are in this case close to the case of the single cylinder, and the discontinuous jumps for critical Reynolds numbers are prominent as well.

For the medium spacing $S / d=2.8$ (the middle plot of Fig. 6), the $S t(R e)$-curve of the downstream cylinder is quite different. In the subcritical Reynolds number range, the Strouhal number is $S t \approx 0.14$ and thus significantly lower than those for the largest distance $S / d=4$. (Alam et al. 2003) obtained nearly the same value. The jump in the supercritical Reynolds number range reaches only $S t \approx 0.34$ and is followed by a stepwise decrease down to a new plateau, the latter beginning at approximately $R e=2 \times 10^{6}$ and having Strouhal numbers somewhat higher than $S t \approx 0.2$. At approximately $R e=8 \times 10^{6}$, there is a distinctive dip down to a level of $S t \approx 0.16$. This dip is coupled with a corresponding dip in the drag coefficient $\mathrm{Cd}_{2}$ in Fig. 4 and in the $\mathrm{Cl}_{r m s}$ of the 
downstream cylinder in the upper plot of Fig. 6. Notably, for this $S / d$-value, the transcritical Strouhal numbers are again close to the subcritical values. (Alam et al. 2003) obtained a value of $S t \approx 0.14$ for the missing subcritical case of $S / d$ $=1.56$, which corresponds to our and Alam's results for $S / d=2.8$.

\subsection{Power spectra}

In the discussion of the behaviour of Strouhal numbers as a function of the Reynolds number, reference was made to the complexity of the underlying power spectra. To give an overview of how the spectra of the fluctuating lift forces $\mathrm{Cl}_{2}(t)$ that act on the downstream cylinder develop from subcritical up to very high Reynolds numbers, twelve spectra, typical for the respective Reynolds number ranges, are shown in Fig. 7.

The selected cylinder spacing is in this case $S / d=4$. In this way, the abrupt changes in the critical range and the appearance of two peaks-with mutual dominance-can clearly be visualised. In the left column of Fig. 8, the most important spectra of Fig. 7 are shown in a two-dimensional representation to allow a more quantitative examination and evaluation.
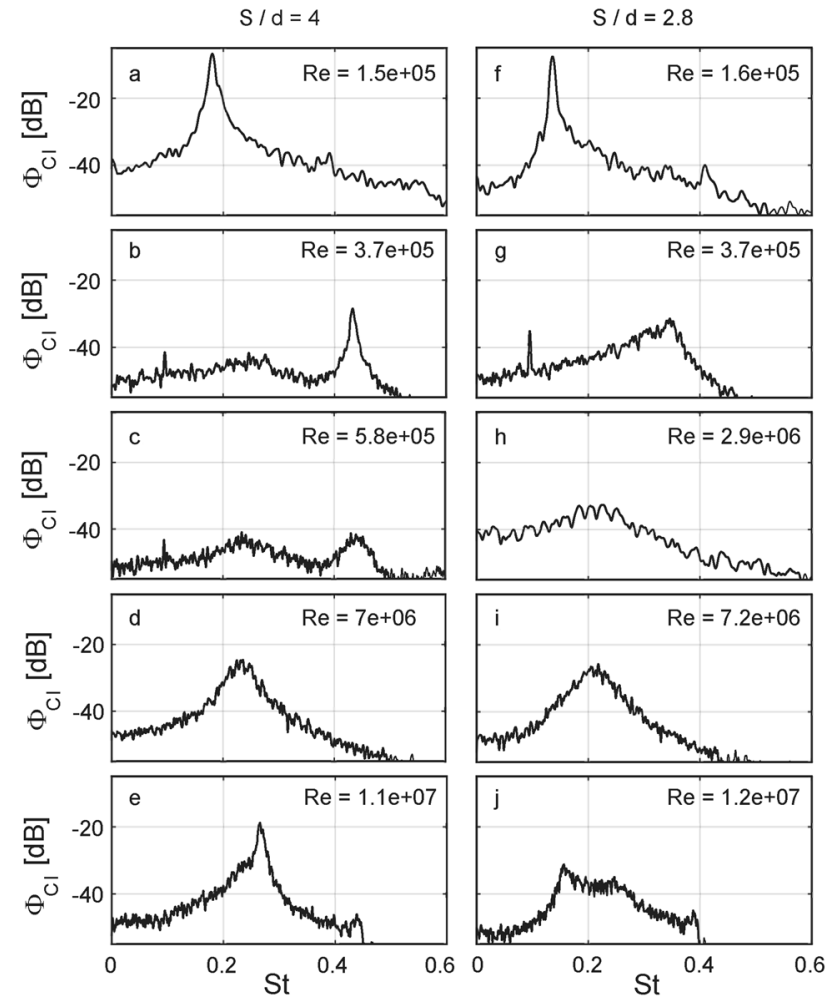

Fig. 8 Power spectra $\Phi_{C l, 2}(S t)$ for different Reynolds numbers, obtained from the fluctuating lift forces $\mathrm{Cl}_{2}(t)$ acting on the downstream cylinder. In the left column, i.e. Fig. 8a-e, the spacing is $S / d=$ 4. In the right column, i.e. Fig. $8 \mathrm{f}-\mathrm{j}$, the spacing is $S / d=2.8$
The lowest grey spectrum in Fig. 7, which corresponds to Fig. 8a, was recorded at $R e=1.5 \times 10^{5}$ and is typical for the subcritical Reynolds number range. The main feature is the single dominant peak at $S t=0.19$, which protrudes more than $35 \mathrm{~dB}$ from the background. The second spectrum (magenta solid line) in Fig. 7 represents the critical state immediately prior to the jump into the supercritical range. The mean drag coefficient is still high (above 1), and the Strouhal number is still low at approximately $S t=0.19$, but in the spectrum, a second smaller peak already appears at $S t=0.42$. In the third spectrum (black solid line) in Fig. 7, which corresponds to Fig. 8b, the jump into the supercritical range has been completed. From the second spectrum in Fig. 7 on, the most striking feature is the appearance of two Strouhal number peaks within the spectrum at the same Reynolds number (see also Fig. 8c). According to Fig. 7, this behaviour is present up to $R e \approx 3 \times 10^{6}$. For larger Reynolds numbers up to $R e=8 \times 10^{6}$, each spectrum consists again of only one broad peak at $S t=0.24$ (see also Fig. 8d). As the Reynolds number increases further, a new and narrower peak develops at a slightly higher Strouhal number of $S t=$ 0.27 , as seen in the last, topmost spectrum in Fig. 7, as well as in Fig. 8e.

In the right column of Fig. 8, Fig. $8 \mathrm{f}-\mathrm{j}$ shows several spectra belonging to the smaller distance $S / d=2.8$. Most of these spectra correspond to the same Reynolds number regimes as their counterparts in the left column so that the influence of the spacing can be seen directly. Except for the lower Strouhal number value at the smaller distance $S / d=$ 2.8 , the shapes of the spectra in the subcritical case (1st row) are similar. Note that for $S / d=2.8$, no spectra with two peaks can be observed. Additionally, in the supercritical case (2nd row), the peak for $S / d=2.8$ is not as pronounced, and the Strouhal number is significantly lower than in the

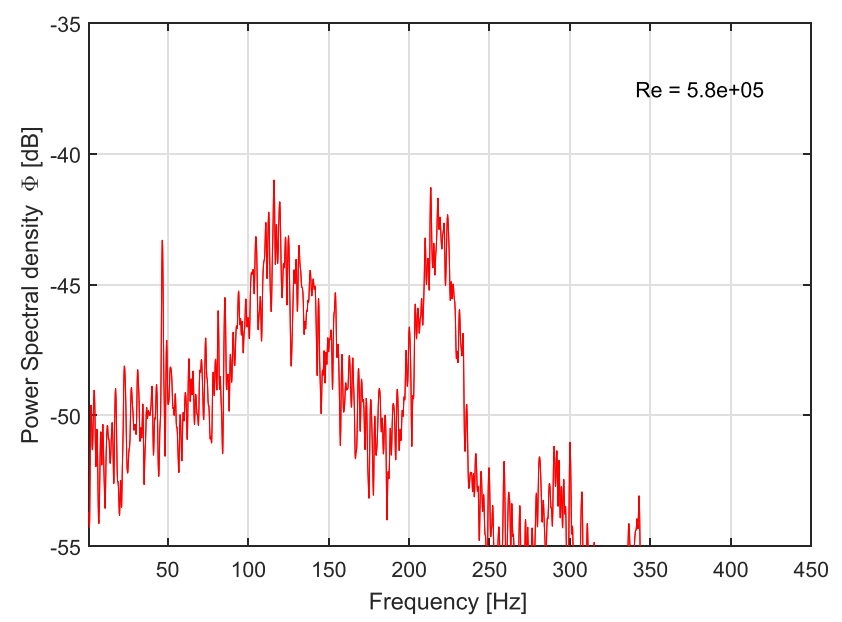

Fig. 9 Power spectrum $\Phi_{C l, 2}(f)$ taken in the supercritical regime (Re $=5.8 \times 10^{5}$ ) for $S / d=4$, corresponding to the spectrum in Fig. $8 \mathrm{c}$, but with horizontal frequency axis and enlarged ordinate scaling 
case of the larger spacing. In Fig. 6, it was already presented that for both distances, the curves for the Strouhal number $S t(R e)$ and the corresponding RMS values of the lift coefficient almost coincide in the range $R e=1 \times 10^{6}-6 \times 10^{6}$. The reason for both observations is the very similar broadband spectra in this Reynolds number range (see Fig. 8d, i). When the Reynolds number continues to rise, a new peak develops from the broadband spectrum, similar to $S / d=4$ (Fig. 8e), but this time at a significantly lower Strouhal number of $S t$ $=0.18$ (Fig. 8j).

Considering all results from the spectral analysis, an important finding is the presence of two peaks in the supercritical region for the larger distance $S / d=4$, which most likely arises from the two different flow modes. Figure 9 shows the special case where the two peaks in the power spectrum $\Phi_{C l, 2}(f)$ for the downstream cylinder have nearly equal heights. The spectrum was taken in the supercritical regime $\left(R e=5.8 \times 10^{5}\right)$ and corresponds to the spectrum shown in Fig. 8c, corresponding to a cylinder spacing of $S / d=4$. To illustrate the effects, the range of the ordinate of the spectrum is slightly adapted, and the abscissa now indicates the frequency.

One broadband peak is present at approximately $f=116$ $\mathrm{Hz}$, and a second relatively narrowband peak is present at approximately $f=218 \mathrm{~Hz}$, the latter corresponding to $S t$ $=0.44$. Both peaks protrude approximately $10 \mathrm{~dB}$ from the spectral background. The ratio of the two frequencies is close to $1: 2$. Hence, subharmonic resonance could play a role here. This possibility is discussed in more detail in sect. 4.2. The (as yet unanswered) question that arises with the usual long-term spectral analysis is in which temporal context the events responsible for the two frequencies occur. By applying the Fourier transform with a sliding window or a wavelet transform, this question can be investigated and is discussed in the following section.

\subsection{Time-frequency analysis-continuous wavelet transform}

When looking at time signals obtained in flows around bluff bodies, the question often rises as to how these signals change as a function of time and what the causes for these changes are. There are two methods to answer these questions: application of a Fourier transform with a sliding window, termed a spectrogram, or a continuous wavelet transform.

We have applied the continuous wavelet transform, which is an alternative to the Fourier transform ( (Gaviria and Montejo 2018)). This algorithm computes the similarity between each segment of a signal and a short, wave-like distribution called a wavelet. The wavelet can be scaled across many widths to capture different frequencies. Due to the variation in the wavelet length, even frequencies far apart from one another can be detected well. In general, the Heisenberg uncertainty principle determines the lower limit for the frequency-time resolution product. The result of a continuous wavelet analysis is then, for example, a "scalogram".

For sub-, super-, and transcritical Reynolds numbers, typical scalograms are presented in the upper parts of Fig. 10a-c. The selected cylinder distance is $S / d=4$. As a contour plot, the scalogram displays the percentage of energy for each wavelet coefficient depending on time $t$ and scale $s$ and is quantified in the colour bar. The scale $s$ is related to the frequency $f$ as $s=F_{s} / f$, with $F_{s}$ being the sample frequency. The complex Morlet wavelet has been applied, which is particularly suitable for oscillatory phenomena. The central frequency is fixed at $F_{c}=1 \mathrm{~Hz}$, and the wavelet width is varied between 2 and 8 .

Before discussing the scalograms, it is suggested to first examine the data on which the wavelet transform is based, namely the corresponding time series of the lift coefficient $\mathrm{Cl}_{2}(t)$ of the downstream cylinder in the lower parts of Fig. 10a, c. They show at first sight a similar appearance to the time series of a single circular cylinder at sub- and transcritical Reynolds numbers (Figs. 13 and 14 in (Schewe 1983)). The corresponding long-term power spectra can be found in Fig. 8a, e. Both time series of the lift coefficient show the appearance of time histories caused by more or less periodic vortex shedding, as is typical for bluff bodies. There is a monofrequency oscillation as the carrier frequency, which is modulated at random.

Returning now to the scalogram of the subcritical case at $R e=1.5 \times 10^{5}$ in Fig. 10a, the rising and falling amplitudes, i.e. the modulation, are reflected in the scalogram by more or less connected islands formed of closed contour lines. These islands or areas are lined up on the horizontal line of scale $s=210$, which corresponds to a vortex shedding frequency of $f=23 \mathrm{~Hz}$. The oval-shaped areas are in the transverse direction aligned to the time axis, and their widths are a measure of the frequency fluctuations due to the vortex shedding process. The inner red centres mark the areas with the largest amplitudes of $\mathrm{Cl}_{2}$, while the white spaces indicate amplitudes close to zero. The latter is particularly evident at $t \approx 1 \mathrm{~s}$, approximately $t \approx 5.5 \mathrm{~s}$, and $t \approx 8.5 \mathrm{~s}$. The scalogram in Fig. 10c describes the transcritical case $(R e=1.1 \times$ $10^{7}$ ). Its appearance is similar to that of the aforementioned subcritical case. However, the main islands or areas are now lined up on the horizontal line of scale $s=36$, which corresponds to a vortex shedding frequency of $f=143 \mathrm{~Hz}$. The corresponding time function shows this typical burst-like character as well, although the degree of randomness regarding the modulation is higher. A special feature is that along the line of scale $s=11$ that corresponds to a frequency of $f$ $\approx 452 \mathrm{~Hz}$, there is a second line of weak oval spots, which occur randomly. This frequency corresponds approximately to the bending frequency of the downstream cylinder in the 

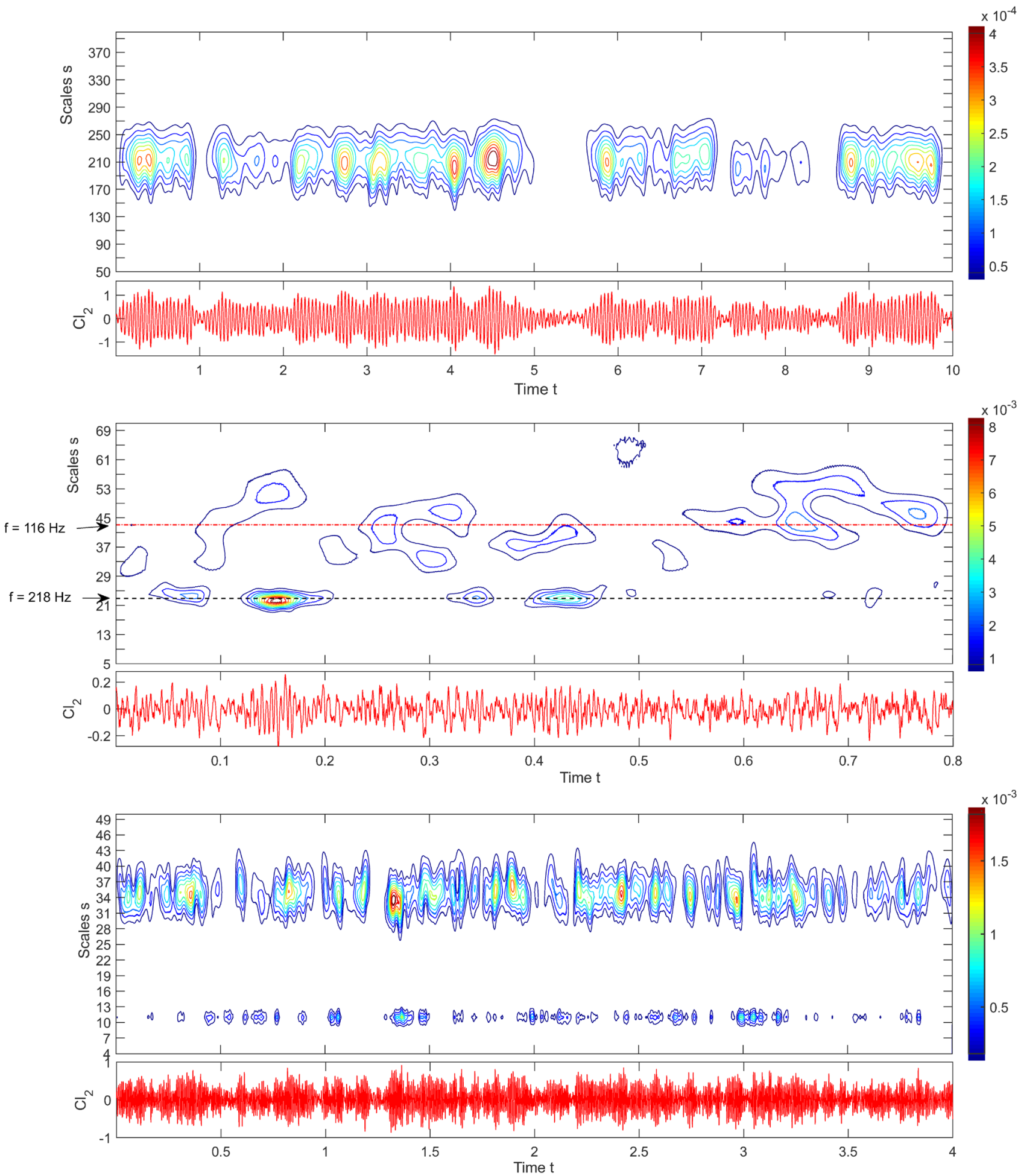

Fig. 10 Wavelet analysis, i.e. "scalogram", of fluctuating lift $\mathrm{Cl}_{2}$ (upper subplots) and the corresponding time function (lower subplots) for typical sub-, super-, and transcritical Reynolds numbers. The scalogram displays the percentage of energy for each wavelet coefficient depending on time and scale $s$ and is quantified in the col- ourbar. Upper image: Subcritical case $\left(R e=1.5 \times 10^{5}\right)$, scale $s=210$ corresponds to $f=23 \mathrm{~Hz}$; centre image: Supercritical case $(R e=5.8 \times$ $\left.10^{5}\right)$; lower image: Transcritical case $\left(\operatorname{Re}=1.1 \times 10^{7}\right)$, scale $s=36 \rightarrow$ $f=143 \mathrm{~Hz} ; s=11 \rightarrow f=452 \mathrm{~Hz}$ 


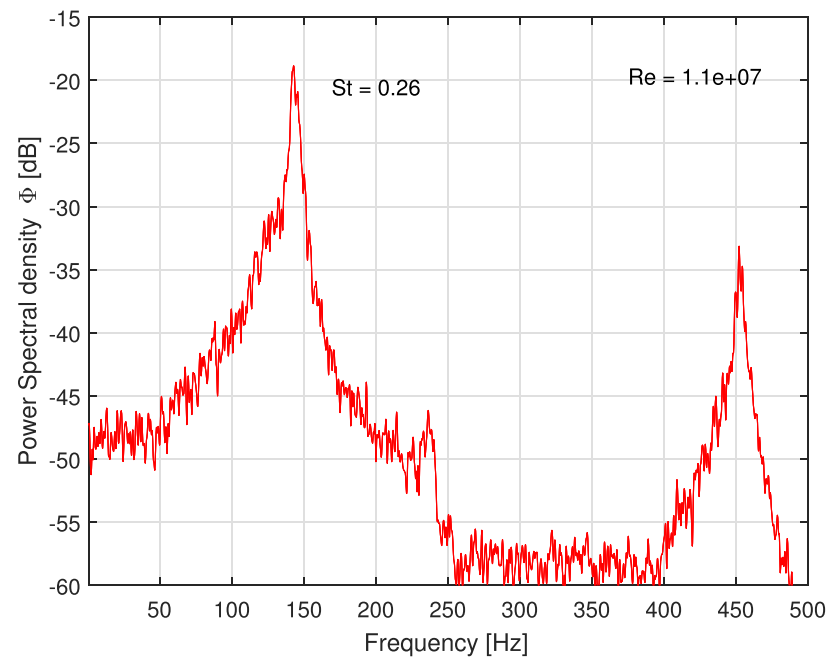

Fig. 11 Power spectrum $\Phi_{C l, 2}(f)$ taken in the transcritical regime at $R e=1.1 \times 10^{7}$, corresponding to the spectrum in Figure 8e, but with extended frequency axis and enlarged ordinate scaling. The peak at $f$ $=452 \mathrm{~Hz}$ is the bending frequency of the downstream cylinder

lift direction. Because of the high pressure $\left(p_{0}=100\right.$ bar $)$ at this Reynolds number, the value is reduced compared to the value of $f_{z}=489 \mathrm{~Hz}$ given in sect. 2.1, which is measured at atmospheric pressure. In this particular case, the bending frequency is slightly excited because its value correlates with three times the vortex shedding frequency at this Reynolds number. This superharmonic resonance of the third order is also visible in Fig. 11. The figure shows the long-term spectrum $\Phi_{C l, 2}(f)$ recorded in the transcritical regime at $R e$ $=1.1 \times 10^{7}$, and apart from the enlarged frequency axis, this spectrum corresponds to the spectrum shown in Fig. 8e.

Figure $10 \mathrm{~b}$ shows the scalogram for a supercritical Reynolds number of $R e=5.8 \times 10^{5}$. This corresponds to the special case where two spectral peaks of nearly equal height are present in the long-term spectrum (see Fig. 9). Overall, the time series has a stochastic character, but there are also intermittently occurring time ranges of approximately $\Delta t \approx 0.1 \mathrm{~s}$ in which burst-like wave packets are detectable. These wave packets are strong enough to produce the two significant peaks in the long time (averaged) power spectrum (Fig. 9). Especially in the time ranges of $t=0.1-0.2 \mathrm{~s}$ and $t$ $=0.4-0.5 \mathrm{~s}$, monofrequency short wave trains appear whose amplitudes vary stochastically. In the scalogram, these burstlike wave packets can be identified as oval elongated areas, aligned inline on the dotted line of scale $s=24$ that corresponds to a frequency of approximately $f=220 \mathrm{~Hz}(S t=$ 0.44 , high-frequency mode). Looking now in more detail at how the peak at approximately $f=120 \mathrm{~Hz}$ is reflected in the scalogram, a slightly different picture is obtained. This frequency corresponds to a scale of $s=41$, and around this dotted line, there are also islands of closed contour lines. However, each of them has a more irregular shape, and compared to the high-frequency mode at $s=24$, the centres of the islands are more widely spread around the line of $s=$ 41. In the time domain, this behaviour is expressed in such a way that there are fewer clear wave trains at $120 \mathrm{~Hz}$. It seems that the low-frequency mode $(s=41)$ is active all the time and that this frequency varies strongly around the average value, whereas the high-frequency mode $(s=24)$ is more intermittent, with a higher degree of regularity and intensity.

The question rises whether the low-frequency mode has something to do with the fact that the cylinder spacing $S / d$ $=4$ is very close to the critical distance $S c$, which could cause the flow to jump back and forth between the two states, namely the "single-body" and "co-shedding" modes ( (Alam et al. 2003)). However, such behaviour would occur at a low frequency, lower than $S t=0.22$, as is the case here. In particular, in a scalogram, such a jump back and forth should be clearly visible. However, in our case, the lowfrequency mode becomes the dominant mode with increasing Reynolds number, indicating that the mode anticipates a flow state characteristic of the very high Reynolds number range before the transcritical regime begins.

\section{Discussion}

\subsection{Flow topology}

(Sumner 2010) described the general flow behaviour around two smooth circular cylinders in a tandem configuration as follows: when two cylinders are arranged inline in tandem, the downstream cylinder is shielded from the incoming flow by the cylinder upstream. The wake of the upstream cylinder modifies the incoming flow conditions for the downstream cylinder, while this second cylinder interferes with the wake dynamics and vortex formation region of the upstream cylinder. This mutual interference means that the upstream cylinder may behave as a generator of unsteadiness, while the downstream cylinder can act as a drag-reduction body, as a target for the impinging shear layers, or as a second vortex shredder. Which of the various interference phenomena described in this way may occur depends mainly on the Reynolds number and the distance between both cylinders. Including the study by (Schewe and Jacobs 2019), a large set of experimental data for three different values of the centreto-centre spacing is now available for sub- to transcritical Reynolds numbers. Since the data were recorded in the same wind tunnel, a comparative discussion is thus appropriate and is given below.

Since no flow visualization was performed in the current study, we have no detailed information on the wake structures around and behind both cylinders, particularly for the super- and transcritical Reynolds number ranges. Nevertheless, we present ideas on how the flow structures 

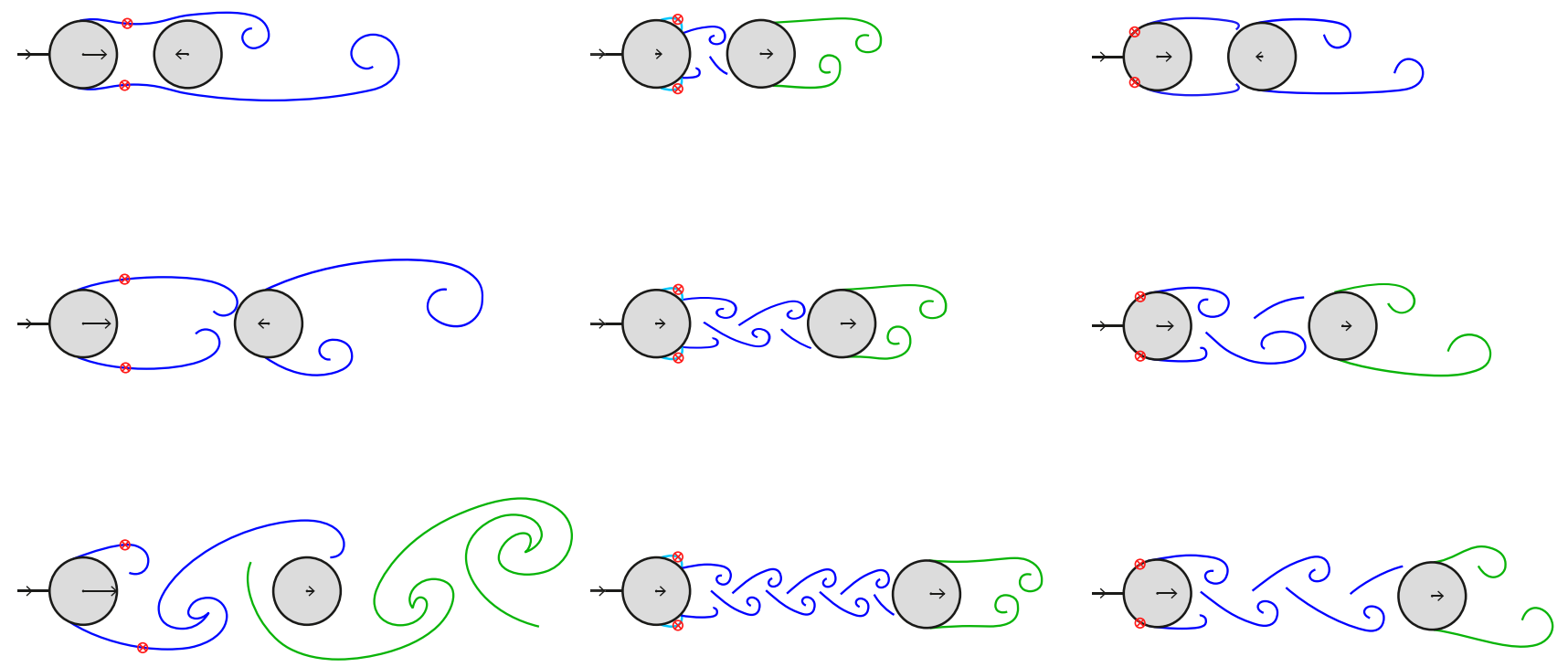

$\otimes$ lam. / turb. transition

Fig. 12 Simplified sketches of the instantaneous 2D flow fields that are typical for the individual Reynolds-number ranges and for three different distances (top: $S / d=1.56$; centre: $S / d=2.8$; bottom: $S / d$ $=4)$. The key role is played by the location of the laminar/turbulent

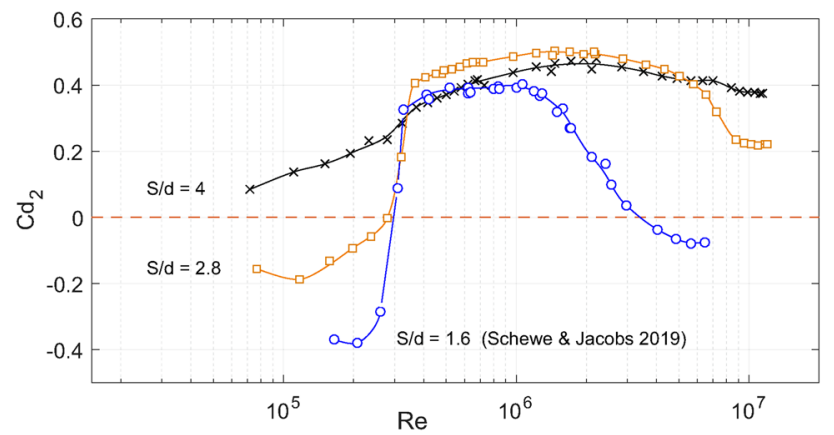

Fig. 13 Mean drag coefficients on the downstream cylinder as function of the Reynolds number for three distances S/d. Apart from the subcritical range for the two smaller distances proximity effects cause a strong dip of $\mathrm{Cd}_{2}$ for Reynolds numbers approaching transcritical values

in the wake could appear, based on our knowledge on the drag coefficients, i.e. $C d_{1}, C d_{2}$, and $C d_{\text {rake }}$, the width of the wake behind the downstream cylinder, and the Strouhal numbers. For this reason, Fig. 12 shows-in a very simplified form-sketches of the flow topologies that are typical for the individual Reynolds number ranges and for the three studied distances $S / d=1.56,2.8$, and 4 . These sketches are intended to represent the instantaneous 2D flow fields, i.e. transition, which wanders upstream for increasing Reynolds number. The vectors indicate the magnitude and direction of the mean drag force coefficients $C d_{1}$ and $C d_{2}$, belonging to the upstream and downstream cylinder, respectively

large-scale vortical structures without consideration of the stochastic (turbulent) nature of the wake flow. They have been arranged in a matrix, in which the individual rows represent the sub-, super-, and transcritical Reynolds number ranges for a constant cylinder interspacing. Each column displays the changes in the flow field for one of these three Reynolds number ranges with varying distance $S$.

Figure 12 demonstrates that the location of the laminar/ turbulent transition (denoted in each sketch by $\otimes$ ) and that the formation of separation bubbles in the supercritical regime play a dominant role regarding the measured Reynolds number effects. Details on the development of the separation bubbles on the cylinder's surface have already been presented in the context of Fig. 4. Starting with the first subcritical case, i.e. the smallest cylinder distance $S / d=1.56$ in the top left image, the separated shear layers undergo transition somewhere in the wake behind the upstream cylinder. The boundary layers separate laminar from the upstream cylinder, and the turbulent free shear layers overshoot the downstream cylinder, as a result of which the two tandem cylinders can be seen as one extended body: "mode I". Because of the presence of a strong negative pressure in the gap between both cylinders, a suction effect is created on the downstream cylinder, resulting in a strong negative mean drag of $C d_{2}=-0.4$ that acts on this cylinder. At this 
small cylinder spacing, the downstream cylinder acts as a drag-reduction body. The wake width behind the downstream cylinder is small, as reflected by a small total drag of $C d_{\text {rake }} \approx 0.6$.

For the second subcritical case, i.e. at a centre-to-centre spacing of $S / d=2.8$ (centre left image), the overall situation is similar. However, as already mentioned in the introductory section, the free shear layers that separate from the upstream cylinder can now reattach to the downstream cylinder, and the flow in the gap remains almost stagnant. This image is an example of the "reattachment regime". Compared with the previous case, i.e. $S / d=1.56$, the larger distance between both cylinders now leads to reduced shielding and diminished suction effects that result in a reduced negative drag force on the downstream cylinder of approximately $C d_{2}=-0.2$. The upstream wake width is further increased, leading to a higher total drag of $C d_{\text {rake }} \approx 1.2$. Both foregoing cases, $S / d=1.56$ and 2.8 , have in common that proximity effects dominate and that one common vortex street is formed behind the downstream cylinder. They are thus both categorised as "mode I".

The last subcritical case at the largest investigated distance of $S / d=4$ (bottom left image) presents an overall situation that has changed fundamentally. This image is inspired by the flow visualization in Fig. 3a in (Alam et al. 2018). Here, both cylinders generate vortices, and the state of the flow is now part of the "co-shedding regime", denoted as "mode II". Because of the large distance, the suction effect on the downstream cylinder has disappeared, which results in a sign change of the drag force on the downstream cylinder; hence, $C d_{2}>0$. The largest distance $S / d=4$ thus lies beyond the critical spacing $S c$, and the downstream cylinder acts as a vortex shredder. Compared with the two aforementioned configurations, the wake behind both cylinders is very broad, which results in the highest total drag among the three cases of $C d_{\text {rake }} \approx 1.7$.

Let us now consider the supercritical cases in the middle column, for which the state immediately after the crossover is sketched. For all three distances, the location of the transition moves upstream with increasing Reynolds number. At the critical Reynolds number, the transition location reaches the shoulders of the upstream cylinder; consequently, separation bubbles form with the laminar/turbulent transition occurring over the bubble. This crossover from the subcritical (left column) to the supercritical (centre column) state is accompanied by a decrease in the wake width behind the upstream cylinder. The separated shear layers produce vortical structures with a characteristic length much smaller than at subcritical Reynolds numbers. This sharp reduction in the characteristic length can be identified from the jumps in the Strouhal number, which are shown in Figs. 6, 8. In addition, the reduction in the wake width reduces the shielding effect and thus causes an increase in the drag $C d_{2}$ on the downstream cylinder. For the two smaller distances, $S / d=$ 1.56 and 2.8 , the transition from subcritical to supercritical Reynolds numbers is accompanied by a sign reversal of $C d_{2}$ to positive drag values, i.e. a crossover from "mode I", where proximity effects dominate, to "co-shedding mode II". It can thus be concluded that a substantial shortening of the critical spacing $S c$ has occurred. For all three cylinder distances in the supercritical range, the mean drag force on the downstream cylinder $C d_{2}$ is positive; hence, all configurations are in the "co-shedding mode". In addition, there is an abnormal situation in which the mean drag forces on the downstream cylinder are higher than those on the upstream cylinder, i.e. $C d_{2}>C d_{1}$. Compared to the three subcritical cases, the downstream wake width at supercritical Reynolds numbers is reduced significantly, which is coupled with a reduction in the total mean drag coefficient to $C d_{\text {rake }}(S / d=$ $1.56) \approx 0.4$ and $C d_{\text {rake }}(S / d=2.8 ; 4.0) \approx 0.6$.

In the third column, the situations with the highest measured Reynolds numbers of $R e \approx 10^{7}$ are outlined. The flow around the upstream cylinder behaves similarly for all three values of cylinder interspacing. The main feature in this transcritical range is the location of the laminar/turbulent transition at the front side of the upstream cylinder. Returning to the upper transition, the simplified process is conjectured as follows: with increasing Reynolds number, the separation bubbles at the upstream cylinder gradually disappear. This process is coupled with an upstream movement of the separation locations. For the smallest of the three distances (top right image), this development is followed by a concurring re-rise of the mean drag force in Fig. 4 and the upstream wake width in Fig. 3. The shielding effect on the downstream cylinder thus increases again and leads to a correlating reduction in the mean drag force on the downstream cylinder. This development continues with increasing Reynolds number until the location of the laminar/turbulent transition reaches the front side of the upstream cylinder and, as a result, overtakes the separation point.

Figure $4 \mathrm{c}$ shows that for the smallest cylinder distance of $S / d=1.56$, the mean drag coefficients of both cylinders level out at a new plateau in the transcritical range. To be more precise, at the beginning of the very high range $(R e \approx$ $3.5 \times 10^{6}$ ), there is a second zero-crossing of the drag of the downstream cylinder to $C d_{2}=-0.2$. Because of this second drag inversion, the state of the flow thus changes once again, this time from "co-shedding mode II" back to the second part of "mode I", the "reattachment mode", in which the free shear layers that have separated from the upstream cylinder can reattach to the downstream cylinder. Compared to the corresponding supercritical state, the total mean drag in the transcritical regime does not change significantly with $C d_{\text {rake }}$ $(S / d=1.56) \approx 0.4$.

For the two other cylinder interspacing values $S / d=2.8$ (centre right image) and 4 (bottom right image), the general 
development is similar to that described above for $S / d=$ 1.56. However, both larger distances, $S / d=2.8$ and 4 , result in weaker proximity effects so that the drag coefficients $C d_{2}$ in both cases remain positive, which means that the critical distance $S c$ is exceeded. The states of the flow in both latter cases thus belong to "co-shedding mode II", in which the downstream wake width is significantly increased, which is then again coupled with an increase in the total mean drag to $C d_{\text {rake }} \approx 0.8$ and 1.2 for $S / d=2.8$ and 4 , respectively.

In the curves in Fig. 13 of the mean drag coefficient on the downstream cylinder, the influence of the proximity effects on the drag force of the downstream cylinder is unambiguous, particularly for the two smaller distances. For the subcritical range and for Reynolds numbers approaching $R e \rightarrow 10^{7}$, the mean drag coefficients decrease significantly, i.e. there is a prominent dip. For $S / d=1.56$, the mean drag force even becomes negative again in the transcritical range.

\subsection{Fluctuating forces}

The discussion of the unsteady effects is unfortunately limited to the two larger distances, since, as has been mentioned before, no unsteady measurements are available for the smallest distance of $S / d=1.56$.

The Strouhal numbers for the largest distance $S / d=4$ in Fig. 6 are very similar to the values for a single cylinder. For Reynolds numbers within the range $R e=1 \times 10^{6}-7 \times 10^{6}$, the spectra of the fluctuating lift for both distances in Fig. 8 are similar, resulting in Strouhal numbers and RMS values of the lift force fluctuations having nearly equal values as well, i.e. $S t \approx 0.22-0.24$. This is followed by branching at higher Reynolds numbers. For $R e \rightarrow 10^{7}$, there appears a dip in the curve of the Strouhal number for $S / d=2.8$ (Fig. 8a) down to $S t=0.17$, as well as a dip in the $C l_{r m s}(R e)$-curve (Fig. 8b). In contrast, for the largest distance $S / d=4$, both curves show increasing trends at high Reynolds numbers. The spectrum in Fig. 8e shows only a single dominant peak at approximately $S t=0.27$ because it can be assumed that a new state has been reached, which remains constant over a larger Reynolds number range. In contrast, the corresponding spectrum for the smaller distance $S / d=2.8$ in Fig. $8 \mathrm{j}$ does not give the impression that a final state has already been reached. The transformation of the spectrum in the range $R e=1 \times 10^{6}$ to $7 \times 10^{6}$ to a new state at higher Reynolds numbers does not seem to have completed yet. There are still remaining peak characteristics that belongs to the previous Reynolds number range, and the new peak at $S t=0.17$ is probably in statu nascendi.

The significance and influence of the interference effects can be determined by comparing the behaviour of the RMS of the fluctuating lift force, $\mathrm{Cl}_{r m s}$, for the tandem cylinder configurations to the case of a single circular cylinder $(S / d$ $=0$ ). Only then does it become clear whether the addition of the second (downstream) cylinder increases or decreases the RMS. For the following comparison, it is once more significant to mention that the experiments for both the single circular cylinder and the tandem cylinder configurations were carried out under almost equal test conditions in the same wind tunnel. The significance of the RMS values of the fluctuating lift is given by the fact that it is an integral value, which reflects the degree of unsteadiness in the entire flow field. Figure 6 shows that for spacing values $S / d=2.8$ and 4, the RMS values on the downstream cylinder are much higher than those for a single cylinder. In particular, at very high Reynolds numbers, approximately $10^{7}, C l_{r m s}$ for $S / d=$ 4.0 reaches values as high as for a single circular cylinder at subcritical Reynolds numbers. At the highest Reynolds number measured for the single cylinder, i.e. $R e=7 \times 10^{6}$, the RMS values for both tandem arrangements are even a factor 4 higher than those obtained for the single cylinder.

In the supercritical range, the most striking feature is the occurrence of two peaks in the long-term spectra for the large distance $S / d=4$ (see Fig. 9). The frequency ratio of the two flow modes is close to $1: 2$. If one considers that in the supercritical Reynolds number range, both cylinders are shedding vortices, i.e. the "co-shedding mode", one can picture them as two interacting fluid oscillators. Typically, these are highly nonlinear and can therefore be responsible for sub- and superharmonic resonances. One could therefore state that the broadband low-frequency mode is due to subharmonic resonance. Nonlinear resonance phenomena in flows around a prism with a square cross section and a Tacoma section were already described in (Schewe 1989).

A further question is whether the flow structures characterised by the two frequencies occur simultaneously or alternately. The wavelet analysis in Fig. 10b shows that (i) the low-frequency mode is more irregular, (ii) it seems to be active all the time, and (iii) the frequency varies strongly around the average value, whereas the high-frequency mode in the form of short wave trains is more intermittent, with a higher degree of regularity and intensity. The scalograms thus provide information on the nature of the fluid forces acting on the body, namely how they change as a function of time. Since the curve of the Strouhal number of the highfrequency mode as a function of the Reynolds number corresponds in its appearance to what is typical for a single cylinder, it can be concluded that the low-frequency mode, which does not occur for a single cylinder, is an interference effect, which is caused by adding a second cylinder up- or downstream.

In this context, it must be emphasized once more that the effect of the fluid forces on the downstream cylinder reflects the unsteady fluctuations of the entire flow field. This means that, even in an attenuated form, there may be contributions in $\mathrm{Cl}_{2}(t)$ - the low-frequency mode-that are mainly produced by events in the flow around the upstream 
cylinder. As mentioned before, this is possible since in the supercritical Reynolds number range, the entire flow field is in a "co-shedding mode"; i.e. both cylinders generate vortical flow structures.

\section{Conclusions}

Based on our measurements regarding Reynolds number effects in the flow around two tandem smooth cylinders at a centre-to-centre spacing of $S / d=2.8$ or 4 , the following conclusions can be drawn:

- Measurements of the steady and unsteady forces acting on a pair of circular cylinders in crossflow for distances $S / d=2.8$ and 4 were performed from subcritical up to ultra-high Reynolds numbers;

- For both distances, the appearance of the drag curves for the upstream cylinder $C d_{1}(R e)$ is similar to the behaviour of a single circular cylinder;

- The drag curves $C d_{2}(R e)$ of the downstream cylinder exhibit the inverse development of that of the upstream cylinder;

- For the subcritical range and for $R e \rightarrow 10^{7}$, the drag on the downstream cylinder decreases significantly; i.e. there is a prominent dip. For $S / d=2.8$, the drag even becomes negative in the subcritical range;

- The drag inversion indicates that the critical distance $S c$ decreases sharply in the supercritical Reynolds number range;

- The curve of the Strouhal number $S t(R e)$ of the downstream cylinder for $S / d=4$ is very close to that of a single cylinder. For the upper transition range at $R e \approx 1 \times$ $10^{6}-7 \times 10^{6}$, the Strouhal numbers lie for both distances between $S t=0.22$ and 0.24 . This is followed by a branching;

- For $R e \rightarrow 10^{7}$ and the case $S / d=2.8$, the Strouhal number dips at $S t=0.17$, whereas the Strouhal number increases up to $S t=0.27$ for $S / d=4$;

- Within the supercritical range, two peaks occur in the power spectra for the larger distance $S / d=4$. Based on a wavelet analysis, we can conclude that the low-frequency mode, which does not occur for a single circular cylinder, is an interference effect.

Acknowledgements The paper is based on a small part of wind tunnel measurements that were performed on behalf of Chevron Corporation and Technip USA, Inc., and accompanied by Jang Whan Kim (Technip USA, Inc.), Bonjun Koo (Technip USA, Inc.), Matthew Kramer (Chevron Corporation), Ho Joon Lim (Technip USA, Inc.) and Guangyu Wu (Chevron Corporation), under the supervision of Jim O'Sullivan (Technip USA, Inc.) and Wei Ma (Chevron Corporation). The authors thank them once more for friendly cooperation and valuable discussions during the tests. The entire test campaign bore the title: Flow around single and tandem cylinders - the effect of the Reynolds number from Re $=10^{4}$ up to $10^{7}$.The authors are also indebted to Markus Löhr (DLRInstitute of Aeroelasticity) and Karsten Steiner (DNW), operators of the High-pressure wind tunnel, to Marc Braune (DLR-Institute of Aeroelasticity) for fruitful discussions during the preparation of this document, and to Christine Unger of the Institute of Aeroelasticity for her support in the creation of various graphics.

Funding Open Access funding enabled and organized by Projekt DEAL. No external funding sources

\section{Declarations}

Conflicts of interest The authors declare that they have no conflicts of interest.

Open Access This article is licensed under a Creative Commons Attribution 4.0 International License, which permits use, sharing, adaptation, distribution and reproduction in any medium or format, as long as you give appropriate credit to the original author(s) and the source, provide a link to the Creative Commons licence, and indicate if changes were made. The images or other third party material in this article are included in the article's Creative Commons licence, unless indicated otherwise in a credit line to the material. If material is not included in the article's Creative Commons licence and your intended use is not permitted by statutory regulation or exceeds the permitted use, you will need to obtain permission directly from the copyright holder. To view a copy of this licence, visit http://creativecommons.org/licenses/by/4.0/.

\section{References}

Alam MM, Moriya M, Takai K, Sakamoto H (2003) Fluctuating fluid forces acting on two circular cylinders in a tandem arrangement at a subcritical Reynolds number. J Wind Eng Ind Aerodyn 91(1-2):139-154

Alam MM, Elhimer M, Wang L, Jacono DL, Wong CW (2018) Vortex shedding from tandem cylinders. Exp Fluids 59(3):1-17

Flachsbart O (1929) From an article by H. Muttray, 1932, Handbuch Experimentalphysik, 4, part 2 (Leipzig), 316

Gaviria CA, Montejo LA (2018) Optimal Wavelet Parameters for System Identification of Civil Engineering Structures. Earthq Spectra 34(1):197-216

Hoerner SF (1958) Fluid-dynamic drag: Practical Information on Aerodynamic Drag and Hydrodynamic Resistance. Published by the Author

Kuzniecow BJ (1931) Aerodinamiczeskije issledowanija cylindrow. Trudy CAGI, wyp. 98, Moskwa-Leningrad (cited in Zuranski, J.,A.: Windbelastung von Bauwerken und Konstruktionen, Verlagsgesellschaft R. Müller, Köln-Braunsfeld, 1969)

Pearcy HH, Cash RF, Salter IJ, Boribond A (1982) Interference effects on the drag loading for groups of cylinders in uni-directional flow. Tech. Rep. R130, Nat. Maritime Inst. Report

Rodriguez I, Lehmkuhl O, Chiva J, Borrell R, Oliva A (2015) On the flow past a circular cylinder from critical to super-critical Reynolds numbers: Wake topology and vortex shedding. Int J Heat Fluid Flow 55:91-103

Roshko A (1961) Experiments on the flow past a circular cylinder at very high Reynolds number. J Fluid Mech 10:345-356

Schewe G (1983) On the force fluctuations acting on a circular cylinder in crossflow from subcritical up to transcritical Reynolds numbers. J Fluid Mech 133:265-285 
Schewe G (1989) Nonlinear flow-induced resonances of an H-shaped section. J Fluids Struct 3:327-348

Schewe G (2001) Reynolds-number effects in flow around more-or-less bluff bodies. J Wind Eng Ind Aerodyn 89:1267-1289

Schewe G (2007) Force and Moment Measurements in Aerodynamics and Aeroelasticity using Piezoelectric Transducers. Springer Handbook of Experimental Fluid Mechanics Springer Handbook, vol 96. Springer, Berlin, pp 596-616

Schewe G, Jacobs M (2019) Experiments on the Flow around two tandem circular cylinders from sub-up to transcritical Reynolds numbers. J Fluids Struct 88:148-166
Sumner D (2010) Two circular cylinders in cross-flow: a review. J Fluids Struct 26(6):849-899

Zdravkovich MM (1987) The effects of interference between circular cylinders in cross flow. J Fluids Struct 1(2):239-261

Publisher's Note Springer Nature remains neutral with regard to jurisdictional claims in published maps and institutional affiliations. 